\section{Retroperitoneal fetus-in-fetu: another supportive evidence for twinning theory}

\author{
Simmi K. Ratan, ${ }^{1}$ Satish K. Aggarwal, ${ }^{1}$ \\ Nita Aneja Khurana, ${ }^{2}$ Anjan Dhua, \\ Gowhar N Mufti, ${ }^{1}$ \\ Vidyanand Deshpandey ${ }^{1}$ \\ ${ }^{1}$ Department of Pediatric Surgery; \\ 2Department of Pathology, Maulana Azad \\ Medical College, New Delhi, India
}

\begin{abstract}
An 8-year-old boy with large abdominal lump causing upper abdominal fullness is presented. Clinically it mimicked a trichobezoar because of the restricted side-to-side mobility of the lump. The lump, however, had calcification and radiological features of fetus-in-fetu (FIF). At laparotomy, a fetiform mass covered with fatty tissue was delivered out of a retroperitoneally located sac (in the bed of stomach). The fetiform mass had an identifiable upper limb bearing digits, one of them also bearing nail. Though histopathological examination did not reveal the presence of vertebral bodies, an advanced differentiation of the bony ends, bearing cartilage qualified the mass as FIF. There was family history of twinning with the elder siblings being dizygotic twins. The origin and diagnostic criteria of FIF are discussed and reference is made to a high likelihood of a twinning as the preceding event in this case.
\end{abstract}

\section{Introduction}

The spectrum of teratoma, fetiform fetus-infetu (FIF), parasitic/acardiac twin and conjoined twins are said to represent early errors of monozygotic twinning. The criteria for FIF and its origin are still controversial. Two popularly forwarded theories for FIF are (i) origin from totipotent cells, akin to that of teratoma (ii) monozygotic twinning resulting in aborted growth of one fetus that gets accommodated in the body of host developing simultaneously. ${ }^{1-3}$ The proponents of the former consider FIF as a highly differentiated benign teratoma. ${ }^{4,5}$ Over the time, the twinning theory is getting more favors, as in FIF the mass is always found enclosed within a sac, somewhat similar to the amniotic sac. In fact, the lining of the sac histologically resembles the amniotic membrane. ${ }^{6}$

We present herein an 8-year-old boy who had FIF in retroperitoneal location. There was also a family history of twinning, with his elder siblings (both girls) being dizygotic twins. The case is being reported in view of the rarity of FIF. Also the history of twinning in the family that lends indirect evidence in favor of theory of twinning for FIF as the event of twinning can repeat in subsequent pregnancies.

\section{Case Report}

An 8-year-old boy presented with a lump in upper abdomen since the age of 2 months. It had been gradually increasing in size. The parents had sought medical advice locally in a remote place, but were advised to wait. He had been complaining of pain for the last one year. Occasional postprandial vomiting was there. The abdominal sonography done elsewhere had shown features suggestive of trichobezoar and the child was referred to our institute for a definitive treatment. The serum AFP and HCG were within normal limits.

At presentation the child had a $15 \times 12 \mathrm{~cm}$ firm well defined non-tender lump extending in left hypochondrium and epigastric region. Restricted side-to-side mobility could be appreciated. Abdominal sonography showed evidence of a well-encapsulated mass in the left lumbar region that had an echogenic centre surrounded by a relatively hypo-echoic area. This hypoechoic area was surrounded by an echogenic rim and had calcification. These radiological signs were suggestive of FIF.,7 Subsequent contrast-enhanced computed tomography (CECT) substantiated these findings (Figure 1). At this juncture, on direct questioning, the mother gave the history of twinning in the previous pregnancy.

At exploratory laparotomy, a $15 \times 20 \mathrm{~cm}$ retroperitoneal tumor was found in the retroperitoneum in the upper abdomen. The pancreas and duodenum were stretched on to the anterior and medial surface of the tumor, respectively, causing substantial difficulty in reaching the tumor. The superior most margin of the tumor reached the left diaphragm but was free from it. The left kidney could be palpated separate from the tumor. The tumor had smooth, comparatively avascular surface with no discrete feeding vessels. The tumor had a large, hard central core that could be moved side-to-side inside the hitherto considered tumor capsule. Since the posterior surface of the tumor appeared to merge imperceptibly in the posterior abdominal wall and the origin of the major blood vessels was not clear, it was decided not to remove the tumor in toto for the fear of jeopardizing the blood supply of gut. The anterior wall of lesion was, therefore, incised and it simulated opening a thick rubber sac. From inside, a fetiform structure wrapped with dense oily layer (simulating vernix caseosa) was delivered out. It was about $15 \times 10 \mathrm{~cm}$ and
Correspondence: Simmi K. Ratan, G-8/6, 2nd Floor Malviya Nagar, New Delhi, India

E-mail: drjohnsimmi@yahoo.com

Key words: fetus-in-fetu, fetiform, twin, trichobezoar.

Received for publication: 6 September 2011. Accepted for publication: 14 October 2011.

This work is licensed under a Creative Commons Attribution NonCommercial 3.0 License (CC BYNC 3.0).

(C) Copyright S.K. Ratan et al., 2011

Licensee PAGEPress, Italy

Teratology Studies 2011; 1:e2

doi:10.4081/ts.2011.e2

weighed about $500 \mathrm{~g}$. The structure was covered all along with skin and simulated a floppy fetus; the head being represented by a globular area bearing hair. No facial features were discernible. An upper limb was identifiable, distal to elbow region, bearing three distinct digits (Figure 2). One of the digits bore a nail. Once the structure was delivered out, the residual cavity was cleaned of the fatty layer covering the fetiform mass. Since, the posterior wall of the sac seemed to merge into the posterior wall of the abdomen imperceptibly; inner lining was excised using innovative scalpel shaving technique after maximally trimming the posterior sac walls within safe limits. The abdomen was closed after lavage.

Histopathological examination shows the mass to be containing derivatives from all three germ layers. No vertebrae could be identified. However, the bones present in the mass had cartilaginous covering suggestive of advanced differentiation (Figure 3 ). The diagnosis of FIF was confirmed histologically.

The child is symptom free at a one-year follow up except for an episode of subacute intestinal obstruction that got resolved with conservative management. Sonographic examination did not reveal any recurrence and his AFP and HCG continue to be within normal limits.

\section{Discussion}

The definition of FIF is full of controversies, as the condition closely resembles teratoma. By and large, the presence of vertebral segmentation, organogenesis or both point to the presence of FIF. ${ }^{2,-10}$ Besides these features, it is important to note that while the growth of FIF commensurate with the growth of the host and stops after reaching a certain size; the teratoma continues to increase in size. The teratoma shows mixture of embryonic and differ- 
entiating tissue at all stages of maturity; ${ }^{2}$ whereas only well organized tissues are present in FIF. For example, teratoma may show tissue differentiation in the form of a segment of intestine and/or calcification in the form of tooth-like structures or even a bony segment but not well developed joints or cartilaginous covering of the bony ends. ${ }^{10}$ The latter are recognized as examples of well-differentiated tissue. Gross examination of the delivered specimen in our patient revealed it to be covered with thick fatty layer (akin to vernix caseosa). Discretely identifiable parts of the upper limb

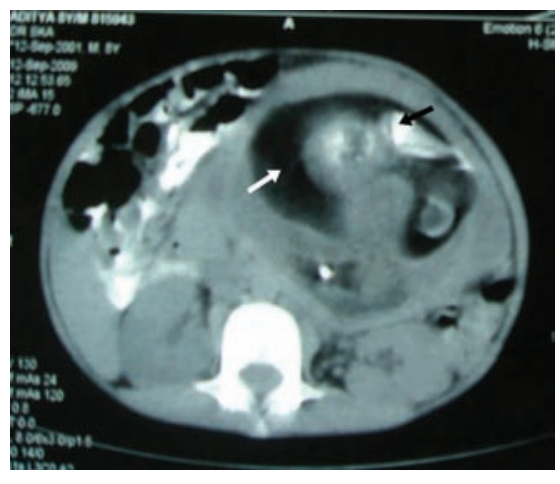

Figure 1. Contrast-enhanced computed tomography of abdomen showing classical finding of echogenic mass in retroperitoneum surrounded by hypo-echoic region (white arrow) and an echogenic rim. The calcification in the mass can be appreciated (black arrow).

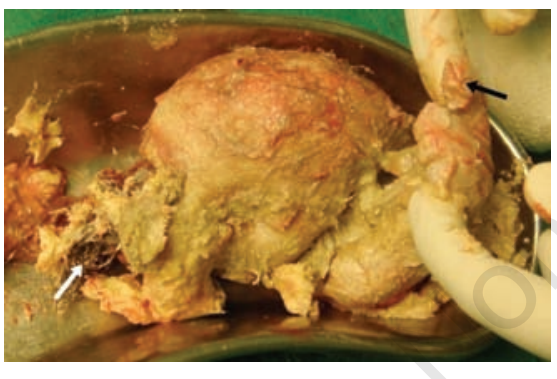

Figure 2. Excised fetiform mass surrounded by vernix caseosa like substance. Globular structure bearing hair (white arrow) and upper limb bearing digits (black arrow) can be appreciated.

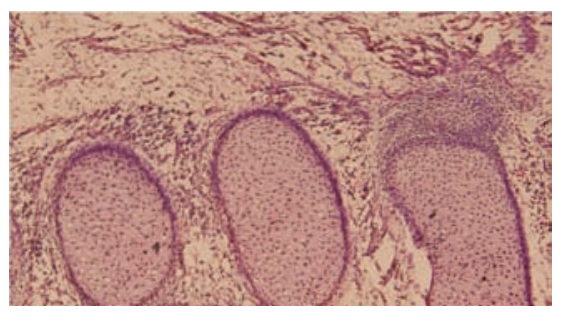

Figure 3. Microscopic examination shows the cartilaginous area over the bones of fetus-in-fetu representing advanced differentiation (x 200). (forearm and hand bearing three digits with nails) could also be recognized. Histopathological examination showed cartilaginous ends of the bones in the digits, and therefore, fits into the definition of FIF.

There is essentially a lack of vertebrae organization in teratoma that vouches for the fact that the pluripotent embryonic tissue escaped the influence of the primary organizer during development. ${ }^{2}$ Therefore, conventionally, the presence of the vertebral segments on gross or microscopic examination is considered to be the cornerstone of definition of FIF. ${ }^{2}$ However, few cases of FIF have been reported in English literature where no vertebral bodies were present, similar to our case. ${ }^{4,10}$ In these reports, the authors label them as FIF with dysplastic vertebral bodies due to the presence of limbs with innervated muscles in them which suggested the existence of spine and the spinal cord (an advanced embryonic differentiation) at some earlier point in gestation. ${ }^{11} \mathrm{Few}$ other authors maintain that the degree of differentiation on histological examination is a better criterion for labeling FIF than the presence of the vertebral bodies. ${ }^{10,12}$ of late, Spencer has proposed that one or more of the following criteria need to be present to label any fetiform mass as FIF: i) Mass needs to be enclosed within a distinct sac. ii) Should be partially or completely covered by normal skin. iii) Should have grossly recognizable anatomic part. iv) Should be attached to the host by only a few relatively large blood vessels. v) Either be located immediately adjacent to one of the sites of attachment of conjoined twins or be associated with the neural tube or the gastrointestinal system. ${ }^{1,2}$ The mass in our patient fulfilled all the above criteria and is the reason for our labeling it as FIF, despite there being an absence of recognizable vertebral bodies.

FIF is generally found enclosed in a retroperitoneal sac (resembling the amniotic sac) and is suspended within the sac with a vascular pedicle; ${ }^{2,13}$ in contrast to a commoner sacrococcygeal location of a teratoma. Besides, teratoma has a broad base, with multiple feeder vessels and continues to grow independently of the growth of the host; whereas the growth of FIF commensurate with that of the host and stops after some time. This observation itself questions the theory of the origin of FIF from totipotent cells and tilts the balance more in favor of twinning theory, especially due to association of FIF with multiple gestations. ${ }^{2}$ In our patient too, a history of twinning in the past pregnancy hinted at repetition of the same phenomenon subsequently and provided indirect evidence supporting the twinning theory. ${ }^{14}$ It seems reasonable to postulate that FIF in our patient resulted when intraembryonic errors initially caused a delayed cleavage of the inner cell mass (leading to monozygotic twinning with two amniotic sacs with single chorionic cavity with probably few inter-amniotic adhesions and a common placenta); and later, an unequal blood supply to the fetuses causing partial incorporation of the under-developing fetus and its chorionic sac into the retroperitoneum of the other fetus (the host, i.e. our patient).

It is interesting to note that FIF and teratoma may co-exist in a single patient, as has been reported by Du Plessis et al. ${ }^{15}$ These authors also relate their finding to the formation of three embryonic masses due to early errors of embryogenesis; which led to the formation of the host, FIF and teratoma in their patient. Such division of inner cell mass is said to be forerunner of twinning/multiple gestation and would have led to delivery of triplets had all these parts received adequate and equal placental supply. But FIF part gets lesser blood supply that aborts its growth and causes its incorporation into the body of the other developing fetus, preferably near the site of vertebral axis, representing the area of notochord in the embryo. It is worth noting that all FIF are anencephalic, due to poor blood supply to the growing brain area., ${ }^{1,8}$

FIF is a benign condition with total cure possible with complete excision. In order to avoid any recurrence, a complete removal of all the membranes is recommended. ${ }^{8}$ This was not possible in our patient due to intimate adherence of the sac with surrounding tissues. A few case reports of occurrence of malignancy following incomplete excision of membranes of FIF have been described. ${ }^{6,8}$ The chances of recurrence in these patients may be due to aberrant rests of placental cells of chorionic sac that get trapped in the body of the host while FIF is getting attached to the host's body since the chorionic villi have been noted at the attachment site of FIF with host. ${ }^{10}$ All the above stated observations in one way or other support the twinning theory for FIF and even its co-existence with teratoma. ${ }^{5,15}$ On the other hand, a well-defined sac for FIF and its limited growth cannot be explained if its origin be traced to totipotent cell. In our patient, neither any teratomatous elements were recognized microscopically in the excised specimen nor was there any raise of AFP and HCG levels in the host's serum. Therefore, chances of recurrence are considered to be negligible in our patient despite an incomplete removal of the chorionic sac. The risk of recurrence can be assumed to be lesser in older children since they have already achieved maximal growth though there could be another peak at puberty. Only a long-term follow up will show the outcome of such patients.In short, a boy with FIF in abdominal location has been described with a view to highlight evidence in favor of twinning theory for genesis of FIF. 


\section{References}

1. Spencer R. Parasitic conjoined twins: External, internal (fetuses in fetu and teratomas), and detached (acardiaes). Clin Anat 2001;14:428-44.

2. Higgins KR, ColeyBD. Fetus in fetu and fetaform teratoma in 2 neonates. $J$ Ultrasound Med 2006;25:259-63.

3 . Willis RA. The borderland of embryology and pathology, $2^{\text {nd }}$ Ed. Washington, DC: Buttersworths; 1962. pp. 442-446.

4. Heifetz SA, Alrabeeah A, Brown BS, Lau H. Fetus in fetu: a fetiform teratoma. Pediatr Pathol 1988;8:215-26.

5. Gilbert-Barness E, Opitz JM, DebichSpicer D, et al. Fetus-in fetu form of monozygotic twinning with retroperi- toneal teratoma. Am J Med Genet A 2003;120A:406-12.

6. Park YJ, Lee DS, Han SJ. Prenatal diagnostic clue for fetus in fetu. Pediatr Surg Int 2007;23:977-80.

7. Brand A, Alves CM, Saraiva C, et al. Fetus in fetu-Diagnostic criteria and differential diagnosis-A case report and literature Review. J Pediatr Surg 2004;39:616-8.

8. Arlikar JD, Mane SB, Dhende NP, et al. Fetus in fetu: two case reports and review of litratrature. Pediatr Surg Int 2009;25: 289-92.

9. Aslanabadi S, Spinner RJ, Zarrintan S, et al. A neonate with cleft palate and a fetal mass in the oral cavity: A rare case of an oral fetus-in fetu. Int $\mathrm{J}$ Pediatr Otorhinolaryngol 2007;71:1617-22.

10. Borges E, Lim-Dunham JE, Vade A. Fetus- in-fetu appearing as a prenatal neck mass. J Ultras Med 2005;24:1313-6.

11. Eng HL, Chuang JH, Lee TY, Chen WJ. Fetus in fetu: A case report and review of the literature. J Pediatric Surg 1989;24: 296-9.

12. Gonzalez-Crussi F. Extragonadal teratomas. Atlas of tumor pathology, $2^{\text {nd }}$ ser, fasc. 18. Washington DC: Armed Forces Institute of Pathology; 1982.

13. Tada S, Yasukochi H, Ohtaki C, et al. Fetus-in-fetu. Br J Radiol 1974;47:146-8.

14. Langman J. Medical Embryology. Part 1. $4^{\text {th }}$ Ed. Baltimore: Williams and Wilkins; 1981. pp 83-101.

15. Du Plessis JP, Winship WS, Kirstein JD. Fetus in fetu and teratoma. A case report and review. S Afr Med J 1974;48:2119-22. 